\title{
Elucidation of Rate Variations for a Diels-Alder Reaction in Ionic Liquids from QM/MM Simulations
}

\author{
Orlando Acevedo ${ }^{\dagger}$ William L. Jorgensen, ${ }^{*, \dagger}$ and Jeffrey D. Evanseck ${ }^{*}, \dot{\dagger}$
}

Contribution from the Department of Chemistry, Yale University, 225 Prospect Street, New Haven, Connecticut 06520-8107 and the Center for Computational Sciences and the Department of Chemistry and Biochemistry, Duquesne University, Pittsburgh, Pennsylvania 15282-1530.

Supporting Material

Scheme S1. OPLS-AA Force Field Atom Types for the Ionic Liquid Ions .....................

Table S1. OPLS-AA Non-Bonded Parameters for the Ionic Liquid Ions........................2

Table S2. OPLS-AA Stretching and Bending Parameters for the Ionic Liquid Ions................2

Table S3. OPLS-AA Torsion Parameters for EMI Cation...................................

Figure S1. Potential energy surface for rotation about the C1-NB-CB-CA2 dihedral angle in the

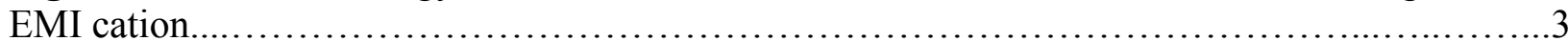

Figure S2. Computed free energy profiles in acidic ionic liquid $\left(\mathrm{EMI}^{+}\right.$and $\left.\mathrm{Al}_{2} \mathrm{Cl}_{7}{ }^{-}\right)$and 1chlorobutane for the reaction between cyclopentadiene and methyl acrylate



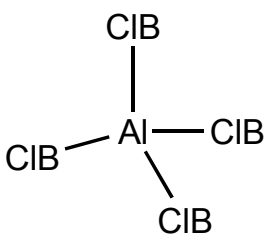

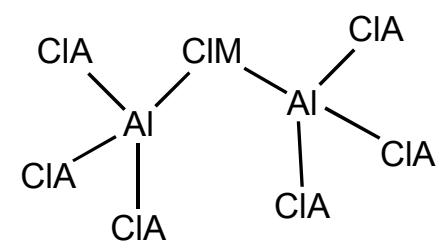

Scheme S1. OPLS-AA force field atom types for the ionic liquid ions. 
Table S1. OPLS-AA Non-Bonded Parameters for the Ionic Liquid Ions.

\begin{tabular}{cccc}
\hline site & $\mathrm{q}, \mathrm{e}$ & $\sigma, \AA$ & $\varepsilon, \mathrm{kcal} / \mathrm{mol}$ \\
\hline C1 & -0.13 & 3.550 & 0.070 \\
C2 & -0.07 & 3.550 & 0.070 \\
NA & 0.16 & 3.250 & 0.170 \\
NB & 0.04 & 3.250 & 0.170 \\
H1 & 0.21 & 2.420 & 0.030 \\
H2 & 0.22 & 2.420 & 0.030 \\
CA1 & -0.22 & 3.500 & 0.066 \\
HA & 0.14 & 2.500 & 0.030 \\
CA2 & -0.11 & 3.500 & 0.066 \\
CB & 0.10 & 3.500 & 0.066 \\
HB & 0.06 & 2.500 & 0.030 \\
Al & 0.72 & 4.050 & 0.100 \\
ClA & -0.37 & 3.770 & 0.148 \\
ClB & -0.22 & 3.770 & 0.148 \\
\hline
\end{tabular}

Table S2. OPLS-AA Stretching and Bending Parameters for the Ionic Liquid Ions.

\begin{tabular}{|c|c|c|}
\hline Bond & $\mathrm{k}_{\mathrm{r}}\left(\mathrm{kcal} \mathrm{mol}^{-1} \AA^{-2}\right)$ & $\mathrm{r}_{\mathrm{o}}(\AA)$ \\
\hline $\mathrm{Al}-\mathrm{Cl} *$ & 126.0 & 2.1771 \\
\hline $\mathrm{C} 1-\mathrm{C} 1$ & 520.0 & 1.360 \\
\hline C1-N* & 427.0 & 1.370 \\
\hline $\mathrm{C} 1-\mathrm{H} 1$ & 367.0 & 1.083 \\
\hline $\mathrm{C} 2-\mathrm{H} 2$ & 367.0 & 1.070 \\
\hline $\mathrm{C} 2-\mathrm{N}^{*}$ & 477.0 & 1.320 \\
\hline NA-CA1 & 337.0 & 1.455 \\
\hline NB-CB & 337.0 & 1.490 \\
\hline CA2-CB & 268.0 & 1.528 \\
\hline CA1-HA & 340.0 & 1.111 \\
\hline $\mathrm{C}^{*}-\mathrm{HB}$ & 340.0 & 1.111 \\
\hline Angle & $\mathrm{k}_{\theta}\left(\mathrm{kcal} \mathrm{mol}^{-1} \mathrm{rad}^{-2}\right)$ & $\theta_{\mathrm{o}}(\mathrm{deg})$ \\
\hline $\mathrm{Cl}^{*}-\mathrm{Al}-\mathrm{Cl}^{*}$ & 12.8 & 109.47 \\
\hline $\mathrm{H} 2-\mathrm{C} 2-\mathrm{N}^{*}$ & 35.0 & 126.0 \\
\hline C2-NA-CA1 & 70.0 & 120.0 \\
\hline CB-NB-C2 & 70.0 & 120.0 \\
\hline CA-NA-C1 & 70.0 & 120.0 \\
\hline CB-NB-C1 & 70.0 & 120.0 \\
\hline NA-CA1-HA & 75.0 & 109.5 \\
\hline NB-CB-CA2 & 100.0 & 110.0 \\
\hline NB-CB-HB & 75.0 & 107.5 \\
\hline NA-C2-NB & 70.0 & 108.0 \\
\hline $\mathrm{C} 2-\mathrm{N} *-\mathrm{C} 1$ & 70.0 & 108.0 \\
\hline CA2-CB-HB & 75.0 & 110.1 \\
\hline HB-CB-HB & 66.0 & 109.0 \\
\hline $\mathrm{C} 1-\mathrm{C} 1-\mathrm{N}^{*}$ & 70.0 & 108.0 \\
\hline C1-C1-H1 & 35.0 & 130.0 \\
\hline $\mathrm{N}^{*}-\mathrm{C} 1-\mathrm{H} 1$ & 35.0 & 122.0 \\
\hline HB-C*-HB & 66.0 & 108.4 \\
\hline HA-CA1-HA & 66.0 & 108.4 \\
\hline
\end{tabular}


Table S3. OPLS-AA Torsion Parameters for EMI Cation.

\begin{tabular}{cccc}
\hline Dihedral & $\mathrm{V}_{1}\left(\mathrm{kcal} \mathrm{mol}^{-1}\right)$ & $\mathrm{V}_{2}\left(\mathrm{kcal} \mathrm{mol}^{-1}\right)$ & $\mathrm{V}_{3}\left(\mathrm{kcal} \mathrm{mol}^{-1}\right)$ \\
\hline C2-NB-CB-CA2 & -0.60 & -1.10 & -0.650 \\
C1-NB-CB-CA2 & -0.60 & -1.10 & -0.650 \\
\hline
\end{tabular}

${ }^{\mathrm{a}}$ For additional dihedral parameters see references $\mathrm{x}$ and $\mathrm{x}$ in the text.

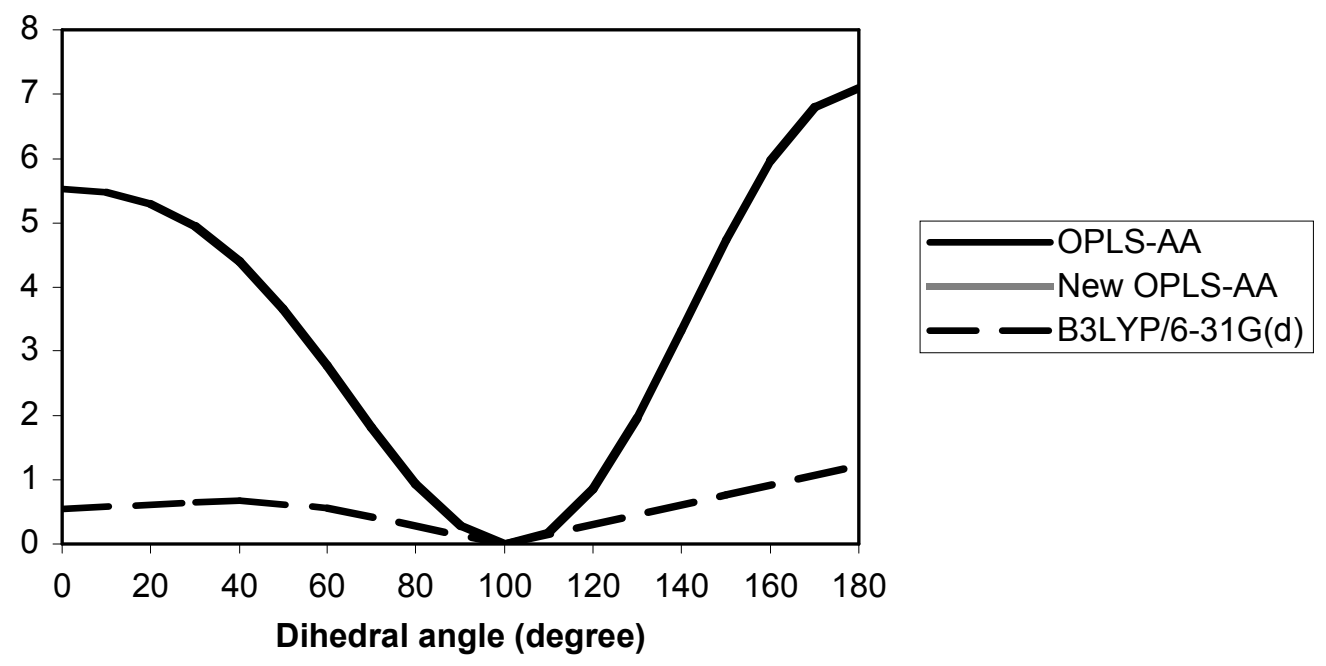

Figure S1. Potential energy surface for rotation about the C1-NB-CB-CA2 dihedral angle in the EMI cation using the original OPLS-AA torsion parameters, new torsion parameters, and B3LYP/6-31G(d). 


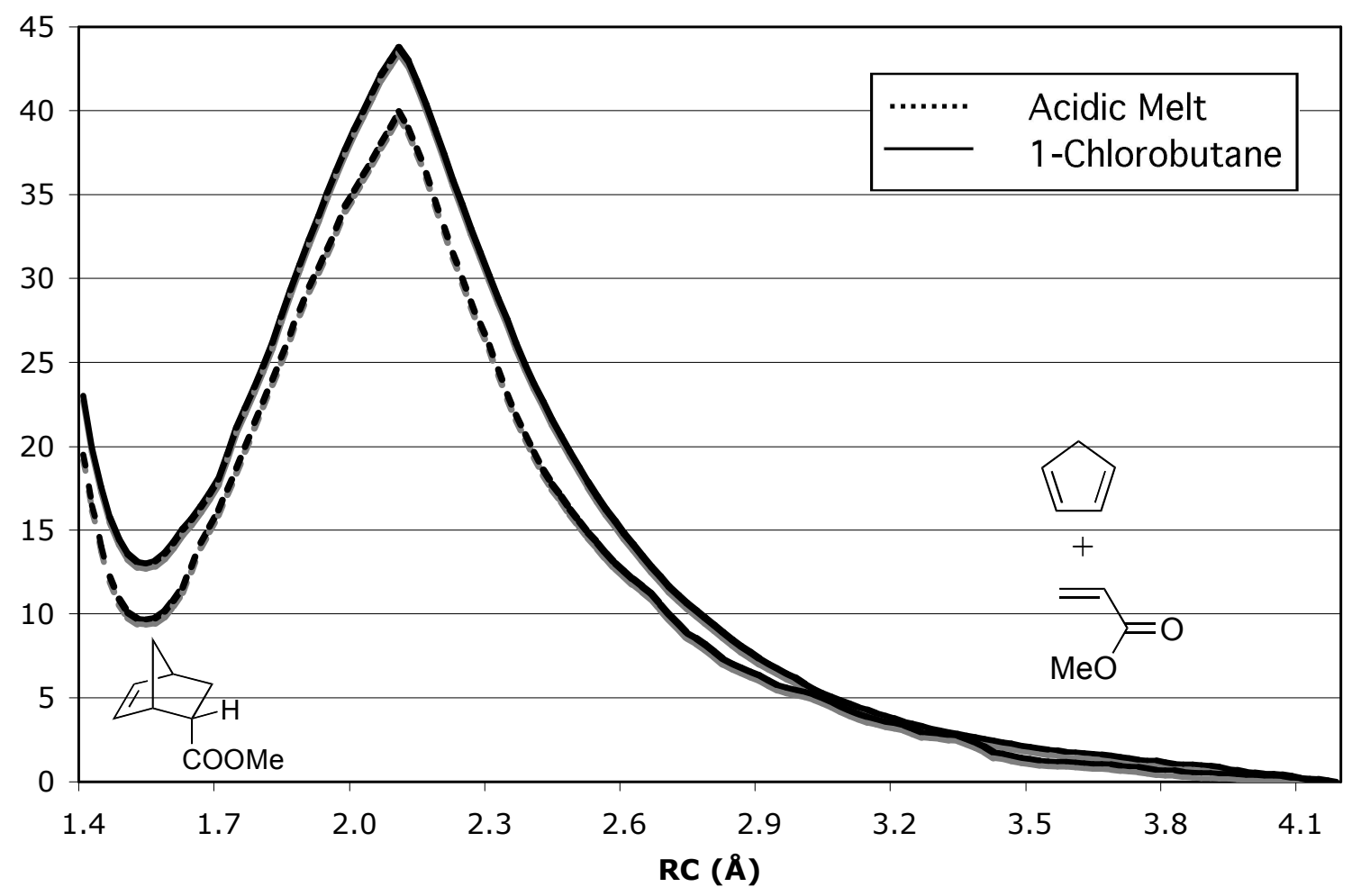

Figure S2. Computed free energy profiles in acidic ionic liquid $\left(\mathrm{EMI}^{+}\right.$and $\left.\mathrm{Al}_{2} \mathrm{Cl}_{7}{ }^{-}\right)$and 1-chlorobutane for the Diels-Alder reaction between cyclopentadiene and methyl acrylate. 